\title{
Communications Transceivers for Venus Surface Missions
}

\author{
Dale A. Force
}

National Aeronautics and Space Administration

Glenn Research Center at Lewis Field

Phone: (216)433-3520; Fax: (216)433-9705

Dale.A.Force@nasa.gov

The high temperature of the surface of Venus poses many difficulties. Previous Venus landers have only operated for short durations before succumbing to the heat. NASA Glenn Research Center conducted a study on communications for long duration Venus surface missions. I report the findings in this presentation.

Current technology allows production of communications transceivers that can operate on the surface of Venus, at temperatures above $450^{\circ} \mathrm{C}$ and pressures of over 90 atmospheres.

While these transceivers would have to be relatively simple, without much of the advanced signal processing often used in modern transceivers, since current and near future integrated circuits cannot operate at such high temperatures, the transceivers will be able to meet the requirements of proposed Venus Surface mission.

The communication bands of interest are High Frequency or Very High Frequency (HF/VHF) for communication between Venus surface and airborne probes (including surface to surface and air to air), and Ultra High Frequency (UHF) to Microwave bands for communication to orbiters.

For HF/VHF, transceivers could use existing vacuum tube technology. The packaging of the vacuum tubes may need modification, but the internal operating structure already operates at high temperatures. Using metal vacuum structures instead of glass, allows operation at high pressure. Wide bandgap transistors and diodes may be able to replace some of the thermionic components. VHF communications would be useful for line-ofsight operations, while HF would be useful for short-wave type communications using the Venusian ionosphere.

UHF and microwave communications use magnetically focused thermionic devices, such as traveling wave tubes (TWTs), magnetron (M-type) amplifiers, and klystrons for high power amplifiers, and backward wave oscillators (BWOs) and reflex klystrons for oscillators. Permanent magnets are already in use in industry that can operate at $500^{\circ} \mathrm{C}$. These magnets could focus electron beam tubes on the surface of Venus. While microwave windows will need to be designed for the high pressure, diamond windows have already been demonstrated, so high-pressure microwave windows can be designed and built. Thus, all of these devices could be useful for Venus surface missions.

Current electronic power conditioners to supply the high voltages used in these microwave devices cannot operate at high temperatures, but earlier electronic power conditioners that used vacuum tubes can be modified to work at high temperature. 
Evaluating the various devices in this study, the M-type traveling wave tube (where a traveling wave structure is used in a crossed-field device, similar to the Amplitron used on the Apollo missions) stood out for the high power amplifier since it requires a single high voltage, simplifying the power supply design.

Since the receiver amplifier is a low power amplifier, the loss of efficiency in linear beam devices without a depressed collector (and thus needing a single high voltage) is not important; a low noise TWT is a possible solution. Before solid-state microwave amplifiers were available, such TWTs were built with a 1-2 dB noise figure. A microwave triode or transistor made from a wide bandgap material may be preferable, if available.

Much of the development work needed for Venusian communication devices will need to focus on the packaging of the devices, and their connections, but the technology is available to build transceivers that can operate on the surface of Venus indefinitely. 